\title{
NOTA CORTA \\ Notas sobre a biologia do minhocoçu Rhinodrilus priollii Righi 1967 em fragmento florestal urbano da Amazônia central, Brasil
}

\author{
Notes about the biology of gigant earthworm Rhinodrilus priollii Righi 1967 \\ in urban forest fragment in the central Amazonia, Brazil
}

INFORMACIÓN

Recibido: 12-03-2016;

Aceptado: 25-06-2016.

Correspondencia autor:

sctcoral@gmail.com
TAPIA-CORAL SANDRA ${ }^{1 *}$ Ph.D, WALDEZ FABIANO² Ph.D.

${ }^{1}$ Grupo de Pesquisa em Biologia, Ecologia e Função de Oligoquetas Terrestres (minhocas e enquitreídeos) Brasileiros. Brasil. ${ }^{2}$ Instituto Federal de Educação, Ciência e Tecnologia do Amazonas (IFAM). Laboratório de Biologia do IFAM Campus Tabatinga, Brasil.
A maioria das minhocas terrestres (Annelida: Clitellata: Oligochaeta) são animais essencialmente edáficos que habitam as camadas superficiais do solo, geralmente até uma profundidade de $50 \mathrm{~cm}$ (BROWN e JAMES, 2007). Nos ecossistemas edáficos as minhocas desempenham importantes funções ecológicas, estando entre os principais componentes dos macroinvertebrados do solo (animais invertebrados visíveis ao olho nu) e pertencendo ao grupo denominado de "engenheiros do solo", em conjunto com cupins (Isoptera) e formigas (Hymnoptera) (LAVELLE et al., 1997). Essa denominação de "engenheiros" decorre desses animais produzirem no ambiente uma considerável quantidade de estruturas físicas que modificam a disponibilidade/acessibilidade de recursos para outros organismos (JONES et al., 1994). As minhocas destacam-se pela capacidade de perfurar o solo e suas galerias criam novos nichos para outros organismos (LAVELLE et al., 1997). A ocorrência das minhocas também aumenta a macroporosidade dos solos, contribuindo para a transformação da matéria orgânica e a mineralização de nutrientes utilizados pelas plantas (STORK e EGGLETON, 1992). As fezes das minhocas são ricas em nutrientes, aumentando, tanto a fertilidade, quanto a biomassa microbiana e a disponibilidade de nitrogênio nos solos (TAPIACORAL et al., 2006).

O termo minhocoçu pela presença do aumentativo "açú" (usu) representa uma palavra herdada da língua indígena Tupi, sendo utilizado para designar as minhocas de grande porte (NAVARRO, 2005). A listagem atual dos minhocoçus do Brasil inclui 53 espécies com tamanhos que variam entre $40 \mathrm{~cm}$ e $200 \mathrm{~cm}$ (BROWN e JAMES, 2007). Esses minhocoçus incluem duas espécies da família Ocnerodrilidae BEDDARD, 1891 e 51 espécies da família Glossoscolecidae MICHAELSEN 1900 (BROWN e JAMES, 2007). As minhocas gigantes têm distribuições restritas, ocorrendo em pequenas áreas geográficas (BROWN e JAMES, 2007). Diversas espécies de minhocoçus encontradas no Brasil têm uma distribuição endêmica e pouco se dispersam das regiões originárias (BROWN e JAMES, 2007; DRUMOND et al., 2013). Essa distribuição restrita, associada ao fato dos minhocoçus não serem criados em vermicultivos, contribui para que espécies de minhocas gigantes sofram processos de extinção em decorrência das mudanças do uso do solo, provocadas pela a agricultura e também, pela captura indiscriminada para abastecer 0 mercado de iscas de pesca (RIGHI e DE MARIA, 1998; BROWN e JAMES, 2007). Como no caso do minhocoçu Rhinodrilus alatus RIGHI 1971 da família Glossoscolecidae, uma espécie endêmica dos cerrados da região central de Minas Gerais no Brasil (DRUMOND et al., 2013), que devido à destruição do seu hábitat e à intensa atividade de coleta para isca foi incluída na lista vermelha das espécies ameaçadas do Brasil, sob a categoria "em perigo de extinção" (RIGHI e DE MARIA, 1998). 
Para a região de floresta Amazônica do Brasil, a maior paisagem natural do pais que engloba cerca de $50 \%$ de sua área, apenas cerca de 15 espécies de minhocoçus, todas da família Glossoscolecidae, foram descritas e distribuídas em cinco diferentes gêneros: Andiorrhinus COGNETTI 1908, Chibui RIGHI e GUERRA 1985, Pontoscolex SCHMARDA 1891, Rhinodrilus PERRIER 1872 e Thamnodrilus BEDDARD 1921 (BROWN e JAMES, 2007; CHRISTOFFERSEN, 2007). Para o estado do Amazonas, o maior estado do Brasil e todo delimitado na região amazônica, apenas cinco espécies de minhocoçus foram registradas: Rhinodrilus elisianae RIGHI et al. 1976, Rhinodrilus lakei MICHAELSEN 1934, Rhinodrilus lucilleae RIGHI et al. 1976, Rhinodrilus priollii RIGHI 1967 e Thamnodrilus salathei MICHAELSEN 1934 (BROWN e JAMES, 2007; CHRISTOFFERSEN, 2007). Uma vez que, relativamente poucas áreas da Amazônia brasileira foram amostradas quanto à diversidade de minhocas (BROWN e JAMES, 2007), tornase evidente a necessidade de maiores estudos que enfoquem na biologia e no manejo dessas espécies na região.

Rhinodrilus priollii RIGHI 1967 é um minhocoçu com ocorrência na Amazônia brasileira que foi descrito pelo pesquisador Dr. Gilberto Righi da Universidade de São Paulo, com base em um único exemplar de $48 \mathrm{~cm}$ procedente das proximidades da cidade de Manaus, no estado do Amazonas, Brasil (RIGHI, 1967). Após sua descrição, RÖMBKE et al. (1999) reportou exemplares de $R$. priollii nos arredores de Manaus em sistemas agroflorestais da Empresa Brasileira de Pesquisa Agropecuária - Embrapa Amazônia Ocidental. Em outro estudo, LANG et al. (2012) encontraram populações de $R$. priollii em quatro diferentes localidades distantes até $70 \mathrm{~km}$ da cidade de Manaus: na fazenda experimental da Universidade Federal do Amazonas (0238'44"S, $60^{\circ} 02^{\prime} 44.20^{\prime \prime} \mathrm{W}$ ), nas estações experimentais do Instituto Nacional de Pesquisas Amazônia (02 $37^{\prime} 33^{\prime \prime}$ 'S, 6002'39' $\mathrm{W}$ ), em uma fazenda privada (02 54 '33"S, 5956'34'W) e no sítio descrito neste estudo. Para os 23 exemplares de $R$. priollii estudados por RÖMBKE et al. (1999) e LANG et al. (2012) foram apresentadas informações sobre a densidade, morfologia clitelar e uma filogenia desses exemplares com base em análise da enzima citocromo c oxidase. Contudo, informações sobre a distribuição e a biologia de $R$. priollii ainda são escassas para as florestas da cidade de Manaus, áreas ameaçadas pelo crescimento urbano. Neste estudo apresentamos dados de biologia para uma população do minhocoçu Rhinodrilus priollii encontrada em um fragmento florestal na cidade de Manaus, Amazonas, Brasil.

A área de estudo, localiza-se na cidade de Manaus, que apesar de estar relativamente isolada em meio à floresta Amazônica do Brasil, representa um gigantesco núcleo urbano que supera os dois milhões de habitantes (BRASIL, 2016a). O fragmento florestal onde foi estudada a população do minhocoçu Rhinodrilus priollii é uma unidade de conservação da categoria reserva particular do patrimônio natural (RPPN), o Centro de Projetos e Estudos Ambientais do Amazonas - CEPEAM (http://cepeam.org.br/), localizado na zona leste da cidade de Manaus (03ㅇ' $06^{\prime}$ "'S, $59^{\circ} 54^{\prime} 17^{\prime \prime} \mathrm{W}$ ), o CEPEAM abriga um fragmento florestal de 55 hectares de floresta secundária com áreas de reflorestamento por espécies nativas, na margem esquerda do rio Negro, na área de encontro com o rio Solimões (Figura 1).
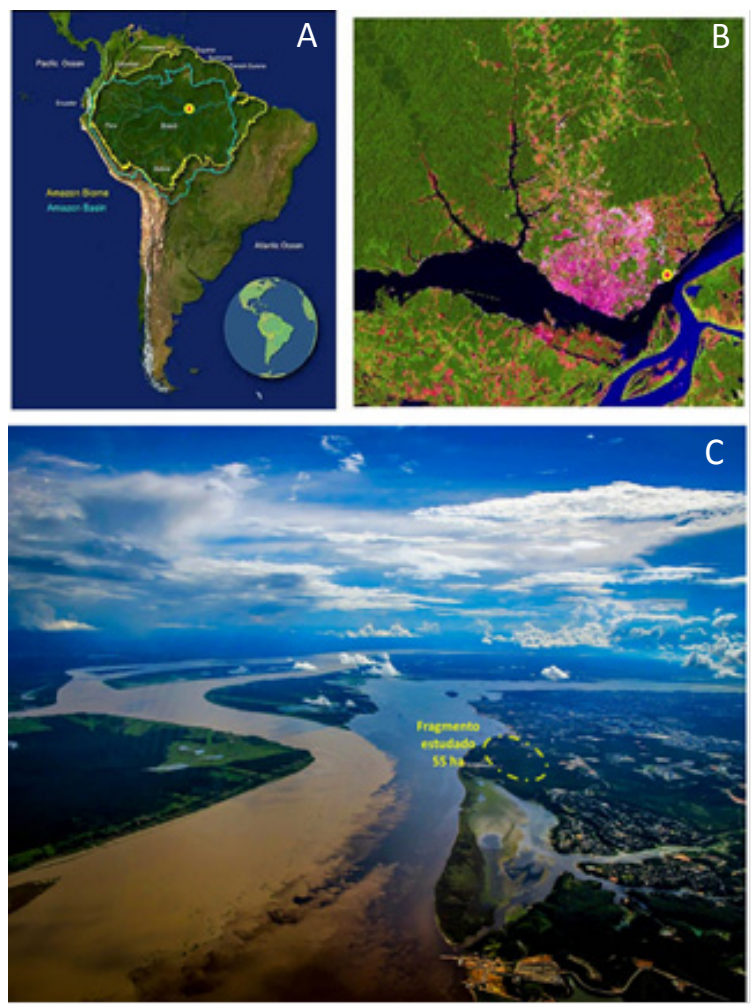

Figura 1. Localização do fragmento florestal onde foram coletados os minhocoçus Rhinodrilus priollii RIGHI 1967, o Centro de Projetos e Estudos Ambientais do Amazonas (CEPEAM) (C), na cidade de ManausAM (B), na Amazônia central, Brasil (A). As imagens foram editadas da INTERNET $\underline{w w w . g o o g l e . c o m . b r ~ e m ~}$ 10/10/2015. 
As coletas aconteceram no período chuvoso, entre março e abril de 2007, quando foram coletados 21 espécimes do minhocoçu Rinodrilus priollii (Glossoscolecidae) na área de floresta secundária do CEPEAM (Figura 2). Vouches dos minhocoçus coletados foram depositados na coleção de invertebrados do Instituto Nacional de Pesquisas da Amazônia em Manaus-AM.

Os minhocoçus foram localizados nas áreas onde se observou grandes aglomerados de fezes (coprólitos) depositados na superfície do solo argiloso da floresta. As coletas ocorreram sempre após uma forte chuva que aparentemente inundou as galerias subterrâneas do solo, obrigando os minhocoçus a saírem para a superfície, onde foram capturados manualmente e colocados em recipientes com solo do local para transporte até o laboratório, onde se procedeu a análise e a identificação dos minhocoçus (Figura 2).
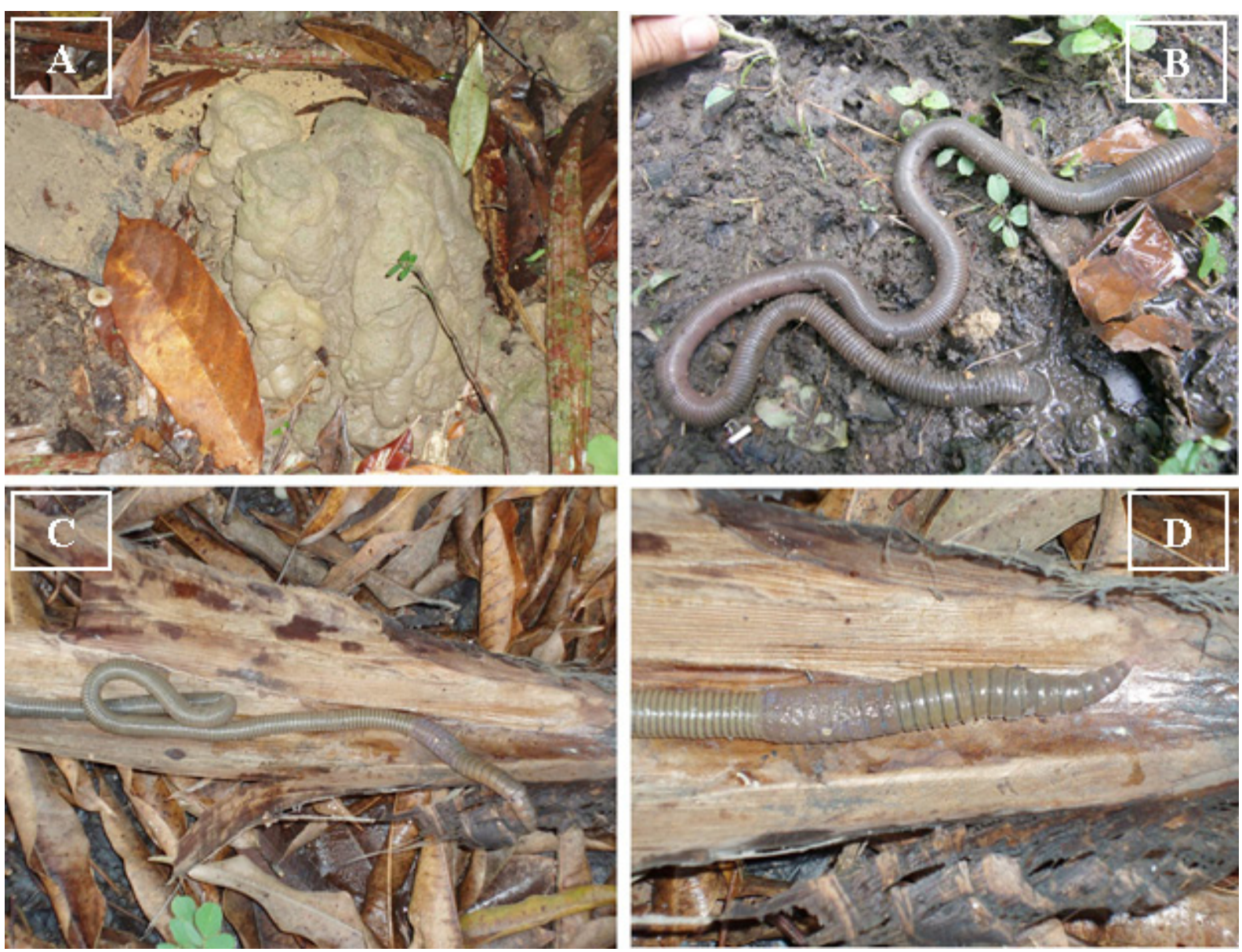

Figura 2. Coprólitos (A) da espécie de minhocoçu Rhinodrilus priollii RIGHI 1967 e indivíduos deslocando-se na superfície do solo da floresta ( $\mathrm{B}$ e $\mathrm{C}$ ) com detalhe para o clitelo desenvolvido de um exemplar sexualmente maduro (D), todos encontrados em Manaus-AM, Brasil.
Uma grande sazonalidade no ciclo de vida foi descrita para outro minhocoçu desse gênero, Rhinodrilus alatus nos cerrados de Minas Gerais, caracterizada por um período de reprodução e forrageamento na estação chuvosa e um período de quiescência na estação seca onde o minhocoçu permanecia enrolado dentro de uma câmera subterrânea (DRUMOND et al., 2013).

O comprimento médio dos $R$. priollii fixados $(\mathrm{N}=$ 21) foi $52 \mathrm{~cm}(D P \pm 11,82)$, variou entre $40 \mathrm{~cm}$ e $75 \mathrm{~cm}$. Os exemplares vivos dessa minhoca gigante mediam até $150 \mathrm{~cm}$ de comprimento, aproximadamente o dobro do tamanho aferido nos espécimes fixados (Figura 3). O peso médio dos minhocoçus fixados $(N=21)$ foi $47 \mathrm{~g}(\mathrm{DP} \pm 12,26)$ e variou entre $28 \mathrm{~g} \mathrm{e} 71 \mathrm{~g}$. 


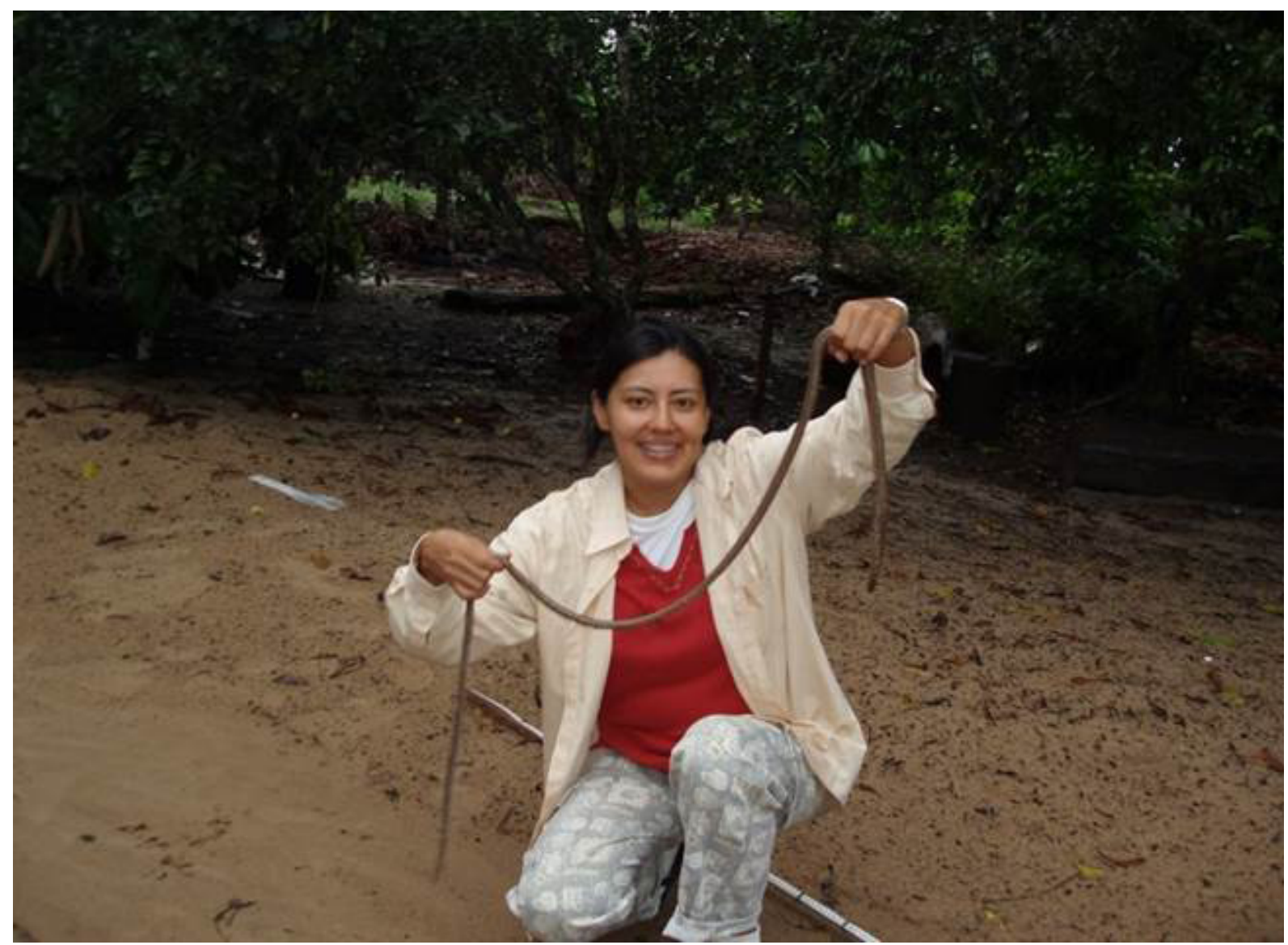

Figura 3. A autora do estudo com um exemplar vivo do minhocoçu Rhinodrilus priollii RIGHI 1967 mostrando o grande tamanho da espécie $(150 \mathrm{~cm})$ capturada em um fragmento florestal da cidade de Manaus-AM, Brasil.

A maioria dos $R$. priollii coletados $(\mathrm{N}=17)$ estavam em fase adulta e maduros sexualmente, apresentando o clitelo desenvolvido com diâmetro médio de $4,31 \mathrm{~cm}(\mathrm{DP} \pm 0,41)$ entre os segmentos corporais 17 e 25 (Figura 2). Acredita-se que o tempo necessário para um minhocoçu alcançar a fase adulta com maturidade sexual esteja entre dois e quatro anos, porém os dados experimentais ainda são raros (DRUMOND et al., 2008).

A taxonomia dos exemplares de Rhinodrilus priollii foi verificada em exemplares adultos e seguiu a descrição de RIGHI (1967), sendo confirmada pelo pesquisador Dr. Samuel James, especialista em minhocas da Universidade de lowa, EUA, através da dissecação dos minhocoçus em laboratório para observação da morfologia interna característica dessa espécie (Figura 4).

Os dados de distribuição do minhocoçu R. priollii apontam que essa espécie está restrita à área de floresta de Manaus, sendo aparentemente limitada pelo rio Negro e distanciando-se até cerca de 70 $\mathrm{km}$ ao norte da cidade (RIGHI, 1967; RÖMBKE et al., 1999; LANG et al., 2012). Portanto, o status de conservação das populações de $R$. priollii pode ser tão preocupante quanto o status de conservação de outros minhocoçus desse gênero do Brasil (RIGHI e DE MARIA, 1998; BROWN e JAMES, 2007; DRUMOND et al., 2012). Desde a implantação da zona industrial na cidade em Manaus em 1967 (Zona Franca de Manaus) (BRASIL, 2016b), esta cidade tem apresentado um rápido crescimento urbano espontâneo que pouco considerou um planejamento ambiental de conservação das áreas de preservação permanente (ex. encostas e margens de corpos hídricos) e também, a interconexão de paisagens entre os poucos parques ecológicos preservados (corredores ecológicos) (CONCEIÇÃO, 2013). De fato, todas as áreas florestais remanescentes em Manaus, encontram-se sob pressão de 

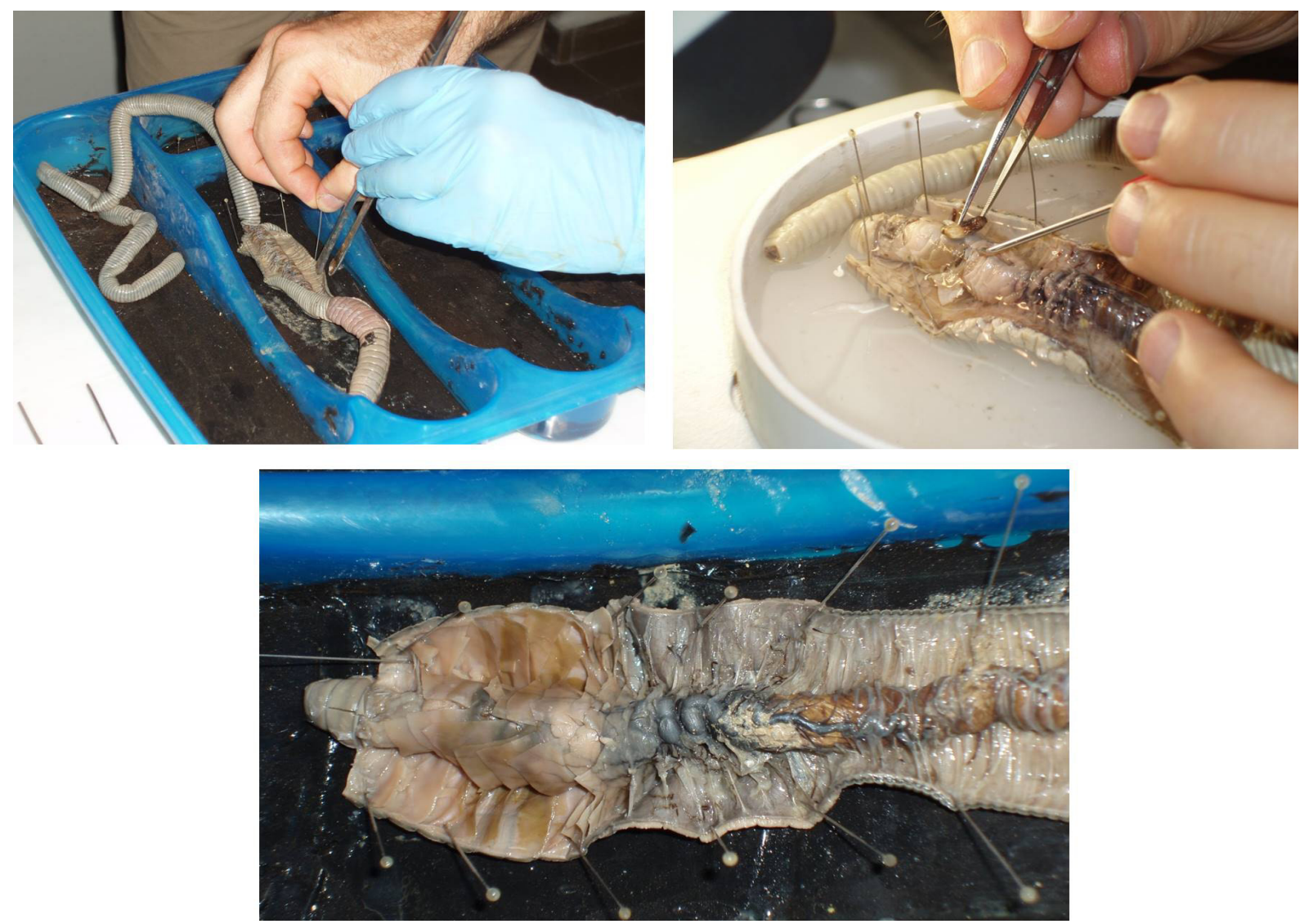

Figura 4. Identificação do minhocoçu Rhinodrilus priollii RIGHI 1967 através de análise da morfologia interna por dissecação dos exemplares no Laboratório de Entomologia do Instituto Nacional de Pesquisas da Amazônia em Manaus-AM, Brasil.

exploração humana, colocando muitas das espécies que possuem distribuição restrita as florestas da cidade sob risco de extinção, como no caso do primata sauim-de-coleira Saguinus bicolor (SPIX 1823) (FARIAS et al., 2015).

Essa situação evidencia a necessidade de avaliar as populações de $R$. priollii nas áreas de florestas onde já foram encontradas, ressaltando a importância desses fragmentos para a conservação dessa minhoca gigante endêmica da Amazônia central brasileira. Além de realizar mais prospecções, a fim de encontrar populações de $R$. priollii em novas áreas também ameaçadas de desflorestamento, pelo novo ciclo de expansão urbana de Manaus, devido a construção de novas vias rodoviárias nessa última década (BRASIL, 2016a).

Agradecimentos: Aos pesquisadores Dr. Jomber Chota Inuma e Dr. Akira Tanaka do Centro de Projetos e Estudos Ambientais do Amazonas (CEPEAM) por terem apoiado logisticamente esse estudo. Ao Dr. Wellington Morais pesquisador do Instituto Nacional de Pesquisas da Amazônia por ter facilitado o acesso ao Laboratório de Entomologia do Instituto Nacional de Pesquisas Amazônia (INPA). 


\section{Referencias}

BRASIL. 2016a. Ministério do Planejamento, Orçamento e Gestão. Instituto Brasileiro de Geografia e Estatística. Disponível em: www.cidades.ibge.gov.brl. Acesso em: ago. 2016.

BRASIL. 2016b. Ministério do Desenvolvimento, Indústria e Comércio Exterior. Superintendência da Zona Franca de Manaus (Suframa). Disponível em: http://site.suframa.gov.brl. Acesso em: ago. 2016.

BROWN, G.G.; JAMES, S.W. 2007. Ecologia, biodiversidade e biogeografia das minhocas no Brasil. Págs. 297-381. Em. Brown, G.G.; Fragoso, C. (Eds.). Minhocas de América Latina: Biodiversidade e ecologia. Londrina: Embrapa Soja. Brasil.

CHRISTOFFERSEN, M.L. 2007. Distribution and species diversity of Rhinodrilus Perrier, 1872 (Annelida, Clitellata, Lumbricina, Glossoscolecidae) in South America. Neodiversity 2:1-6.

CONCEIÇÃO, B.S. 2013. Padrões de ocorrência de aves de floresta como indicadores para a identificação de fragmentos florestais urbanos prioritários para a conservação em Manaus. Dissertação de Mestrado. Programa de Pós-Graduação em Gestão de Áreas Protegidas na Amazônia - MPGAP. Instituto Nacional de Pesquisas Amazônia, Brasil.

DRUMOND, M.A.; CAMPOS, S.H.; GUIMARÃES, A.Q.; NUNES, J.T. 2008. Uso e conservação do minhocuçu Rhinodrilus alatus. MG-Biota 1 (3):5-23.

DRUMOND, M.A.; BROWN, G.G.; MARINI-FILHO, O.J. 2012. Avaliação do risco de extinção do minhocuçu Rhinodrilus alatus RIGHI 1971. Biodiversidade Brasileira 2 (2): 134-139.

DRUMOND, M.A.; GUIMARÃES, A.Q.; EL BIZRI, H.R.; GIOVANETTI, L.C.; SEPÚLVEDA, D.G.; MARTINS, R.P. 2013. Life history, distribution and abundance of the giant earthworm Rhinodrilus alatus RIGHI 1971: conservation and management implications. Brazilian Journal of Biology 73 (4):699-708.

FARIAS, I.P.; SANTOS, W.G.; GORDO, M.; HRBEK, T. 2015. Effects of Forest Fragmentation on Genetic Diversity of the Critically Endangered Primate, the Pied Tamarin (Saguinus bicolor): Implications for Conservation. Journal of Heredity 106: 512-521.

JONES, C.G.; LAWTON, J.H.; SHACHAK, M. 1994. Organisms as ecosystem engineers. Oikos 69:373-386.

LANG, S.A.; GARCIA, M.V.; JAMES, S.W.; SAYERS, C.W. 2012. Phylogeny and clitellar morphology of the giant Amazonian earthworm, Rhinodrilus priollii (Oligochaeta: Glossoscolecidae). American Midland Naturalist 167:384-395.

LAVELLE, P.; BIGNELL, D.; LePAGE, M.; WOLTERS, V.; ROGER, P.; INESON, P.; HEAL, O.W.; GHILLION, S. 1997. Soil function in a changing world: the role of invertebrate ecosystem engineers. European Journal of Soil Biology 33:159-193.

NAVARRO, E.A. 2005. Método moderno de tupi antigo: a língua do Brasil dos primeiros séculos. $3^{a}$ edição. São Paulo. Global. São Paulo, Brasil.

RIGHI, G. 1967. Descrição de Rhinodrilus priollii, sp. n. Glossoscolecidae da Amazonia com bibliografia dos Oligochaeta terricola da região. Atas do Simpósio sobre a Biota Amazônica, 5 (Zoologia). Rio de Janeiro: Conselho Nacional de Pesquisas:475-479. 
RIGHI, G.; DE MARIA, M. 1998. Rhinodrilus alatus RIGHI 1971. Págs. 579-581. Em: Machado, A.B.M.; Fonseca, G.A.B.; Machado, R.B.; Aguiar, L.M.; Lins, L.V. (Eds). Livro vermelho das espécies ameaçadas de extinção da fauna de Minas Gerais. Belo Horizonte: Fundação Biodiversitas. Brasil.

RÖMBKE, J.; MELLER, M.; GARCIA, M. 1999. Earthworm densities in central Amazonian primary and secondary forests and polyculture forestry plantation. Pedobiologia 43:518-522.

STORK, N.E. e EGGLETON, P. 1992. Invertebrates as determinants and indicators of soil quality. American Journal Alternative Agriculture 7:38-47.

TAPIA-CORAL, S.C.; LUIZÃO, F.J.; BARROS, E.; PASHANASI, B.; DEL CASTILLO, D. Effect of Pontoscolex corethrurus MULLER 1857 (Oligochaeta: Glossoscolecidae) inoculation on litter weight loss and soil nitrogen in mesocosms in the Peruvian Amazon. Caribbean Journal of Science 42 (3):410-418. 